\title{
ARTIFACT CHANGES IN THE ULTRASTRUCTURAL FEATURES OF CELLS AND TISSUES DUE TO PREPARATORY PROCEDURES
}

\author{
Atsushi Ichikawa and Misao Ichikawa \\ Department of Anatomy. Yokohama City University School of Medicine
}

\begin{abstract}
Abstrect: A variety of morphological changes in cells and tissues due to preparative procedures were compared at the electron microscopic level using specimens processed by immersion- and perfusionmethods of chemical fixation as well as quick freezing followed by freeze-substitution. The reality or artifact of some observations obtained from the materials processed by conventional chemical fixation, such as the so-called "intercellular interdigitation" of neighboring epithelial cells, open appearance of the lumen in the renal proximal convoluted tubules, the cytoarchitecture and secretory process of mucussecreting cells, the morphological process of fusion and fission of biological membranes and others, is discussed by comparing the morphological observations obtained from the materials processed by conventional chemical fixation to those processed by quick freezing followed by freeze-substitution. The latter method is considered to be the most effective fixation method for preserving the cellular structures more closely in their living state. (J Toxicol Pathol 2 : 15/ 161, 1989)
\end{abstract}

Key words: Fixation artifact, Chemical fixation, Quick freezing. Freeze-substitution

\section{Introduction}

There is no doubt about that the precise diagnosis of pathological changes in cells and tissues is possible to be made only by correct understanding of normal structural features of the cellular elements in their living state. It is wellknown that in conventional chemical fixation, chemical fixatives employed should be prepared to adjust their $\mathrm{pH}$ and osmolarity to those of the tissue to be examined to prevent artifacts. However, it may still cause artifactitious alterations of some structural features of cells and tissues, such as deformation or disruption of membrane-limited structures, swelling, shrinkage, precipitation, and/ or extraction of soluble macromolecules. Recent advance in cryofixation techniques, especially quick freezing, has made it possible to minimize these artifactitious alterations caused by conven-

市川 7 市川

Accepted for publication : August 8, 1989

Mailing address: Atsushi Ichikawa, Department of Anat-

omy, Yokohama City University School of Medicine, 3-9

Fukuura, Kanazawa-ku, Yokohama 236, Japan. tional chemical fixation ${ }^{1-3}$. Cryofixation in which fresh tissue samples are quickly frozen within a few milliseconds is advantageous not only to preserve the structural features of cells and tissues in their living state but also to capture transient physiological processes as evanescent as fusion and fission of membranes, ${ }^{4,5}$. The cryotechnique is also superior to chemical fixation for retaining soluble components in cells, which may be extracted during preparatory procedures of conventional chemical fixation. This allows us to examine the precise localization of these materials by histochemical and immunocytochemical methods $^{3.0}$ as well as autoradiography ${ }^{7}$. In this paper, some examples of artifacts caused by conventional chemical fixation are demonstrated in the acinus of gerbil parotid and sublingual glands, the renal tubules of rat kidney, mucus-secreting cells (goblet cells) in mouse colonic mucosa, and mouse pancreatic exocrine cells.

\section{Materials and Methods}

Male adult Mongolian gerbils (Meriones unguiculatus) weighing $45-80 \mathrm{~g}$, male adult Wistar 
rats, $230-265 \mathrm{~g}$ in body weight, and male adult $\mathrm{C} 3 \mathrm{H}$ mouse weighing 25 to $32 \mathrm{~g}$ were used in this study. They were maintained on standard rat food pellets and water ad libitum and fasted for $12 \mathrm{~h}$ before sacrifice. The pancreatic tissue was obtained from the mouse $60 \mathrm{~min}$ after refeeding after fasting overnight. For conventional immersion method of chemical fixation, small tissue blocks of the major salivary glands. kidney and colonic mucosa were excised under nembutal anesthesia, and immersed in $2.3 \%$ glutaraldehyde in phosphate buffer (pH 7.2. $980 \mathrm{mOsmol}$ ) kept at $4^{\circ} \mathrm{C}$ for $24 \mathrm{~h}$. Perfusion fixation was carried out by infusion of $2.3 \%$ glutaraldehyde in $\mathrm{Na}$-cacodylate buffer ( $\mathrm{pH} 7.2,320 \mathrm{mOsmol}$ ) kept at $37^{\circ} \mathrm{C}$ into the abdominal aorta ${ }^{a}$, and $10 \mathrm{~min}$ after initiation of infusion tissue blocks were excised, cut into small blocks and immersed in the same fixative kept at $4^{\circ} \mathrm{C}$ for $12 \mathrm{~h}$. Those samples processed by immersion method as well as perfusion method were washed in buffer, postfixed with $1 \% \mathrm{OsO}_{4}$ in buffer, dehydrated in a series of graded concentrations of cold ethanol and embedded in an epoxy resin mixture after replacing alcohol with propylene oxide. For cryofixation, fresh tissue blocks were excised under nembutal anesthesia, quenched by smashing against the polished surface of a pure copper block precooled with liquid helium and transferred into liquid nitrogen'. They were then substituted with $4 \%$ OsO, in acetone kept at $-80^{\circ} \mathrm{C}$ for $36 \mathrm{~h}$, rinsed in 3 changes of acetone for $5 \mathrm{~min}$ each, trimmed in acetone so as to expose the area frozen first by contact with the metal block, and embedded in an epoxy resin rixture after minsing in propylene oxide. Thin sections were obtained with Sorval MT-I ultramicrotome, mounted on copper grids, stained with uranyl acetate and lead citrate, and examined with a JEOL $100 \mathrm{CX}$ electron microscope. For light microscopy, semithick sections were stained with toluidine blue.

\section{Results and Discussion}

Intercellular interdigitation: The lateral boundaries of neiboring cells in the serous acinus of gerbil parotid gland are often seen to be interdigitated with each other after conventional chemical fixation (Fig. 1). This is generally interpreted to be one of the intercellular contacts to show a

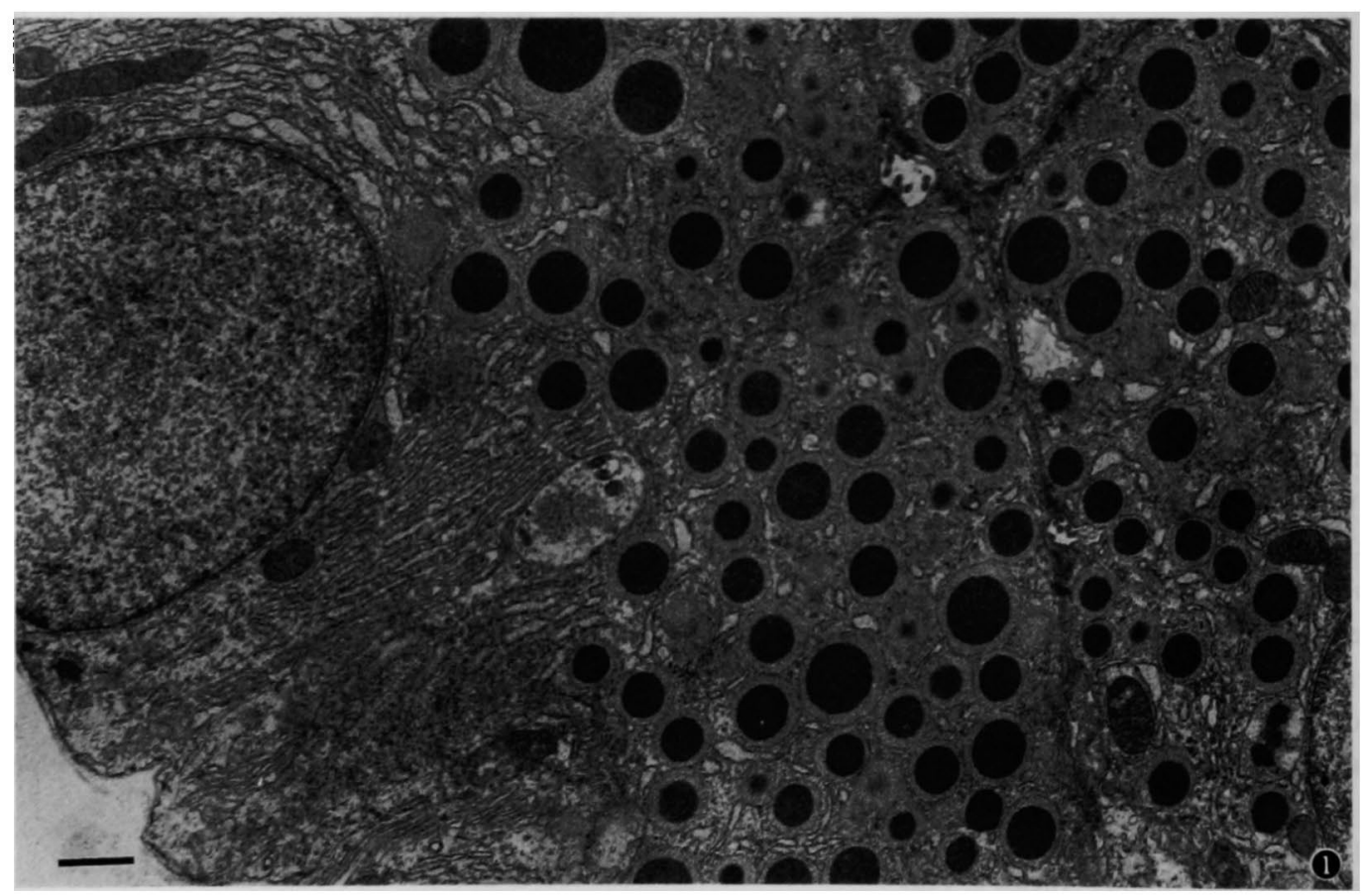

Fig. I. Serous acinar cells of gerbil parotid gland processed by perfusion fixation with glutaraldehyde buffer-solution followed by postosmication. Intercellular space appears to be occluded and the lateral plasma membranes are partly interdigitated. $\times 7,900$. 


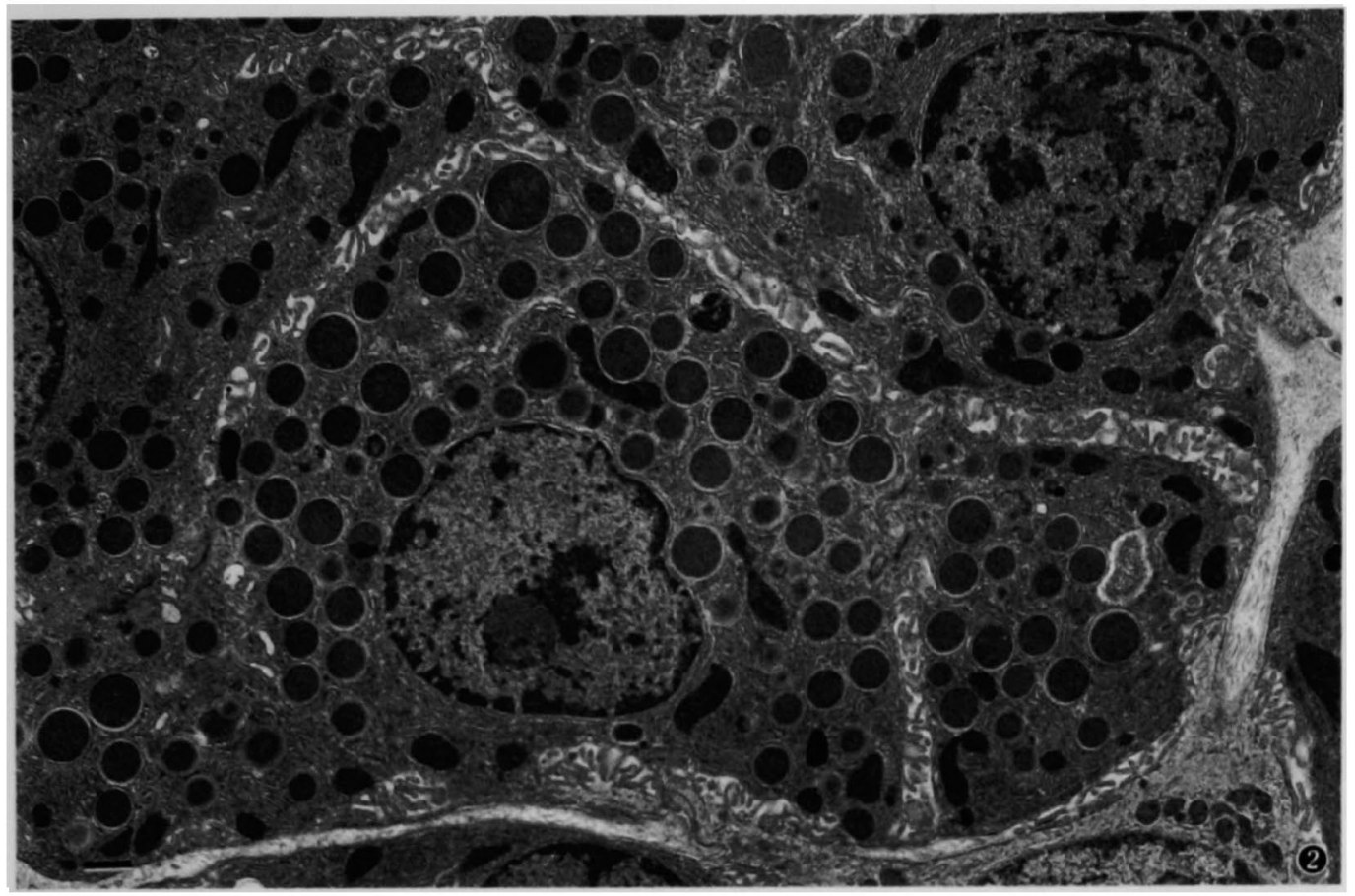

Fig. 2. Parotid acinar cells of gerbil processed by quick freezing followed by freeze-substitution. Intercellular space is open with a certain distance and filled with numerous cell folds of apposed acinar cells. $\times 5,000$.

close relationships between the cells. Similar findings are observed in other epithelia such as the colonic mucosa, duct epithelia of the major salivary glands, mucous membrane of the gall-bladder and others. However, after primary cryofixation, the lateral borders of these epithelial cells are seen to be separated from each other by a space $520-750$ $\mathrm{nm}$ in width which is filled with an interdigitation of regularly arranged, numerous cell folds (Fig. 2). These intercellular space is known to be physiologically active as a pathway of ions and nutrients absorbed ${ }^{-11}$. It seems likely that the broad intercellular space provided with amplification of cell surface area is more advantageous for such functions, and the observation found in cryofixed materials has a reality.

Morphological changes in the proximal convoluted tubule of rat kideney due to specimen preparations: As seen in figures $3 a$ and $3 b$, the lumen of the proximal convoluted tubule appears to be occluded by the apposition of the apical surfaces of the surrounding cells in the materials processed by immersion method of chemical fixation, while in those processed by perfusion fixation all the proximal tubules are seen to have a wide open lumen. This has been reported by Maunsbach in 1966'12. It is also known that if osmium fixative is dripped onto the surface of the living kidney covered with the peritoneum the proximal tubules in the superficial layers of the renal tissue appear to have an open lumen. If fresh tissue blocks of the renal tissue are processed by quick freezing followed by freeze-substitution, the tubular lumen appears to be open with only a narrow space (Fig. 4). It was initially considered that obliteration of the tubular lumen might be advantageous for absorption of some elements of the glomerular filtrate through the brush boaders, but it is now believed that the proximal tubules have a wide open lumen under normal condition because of the observaton of an open lumen obtained by perfusion and/or dripping methods. Closed appearance of the tubular lumen in the materials processed by conventional immersion method of chemical fixation may be attributed to swelling of the epithelial cells caused during fixation, because the luminal plasmamembrane of the epithelial cells is frequently seen to be swollen up as greatly as to 

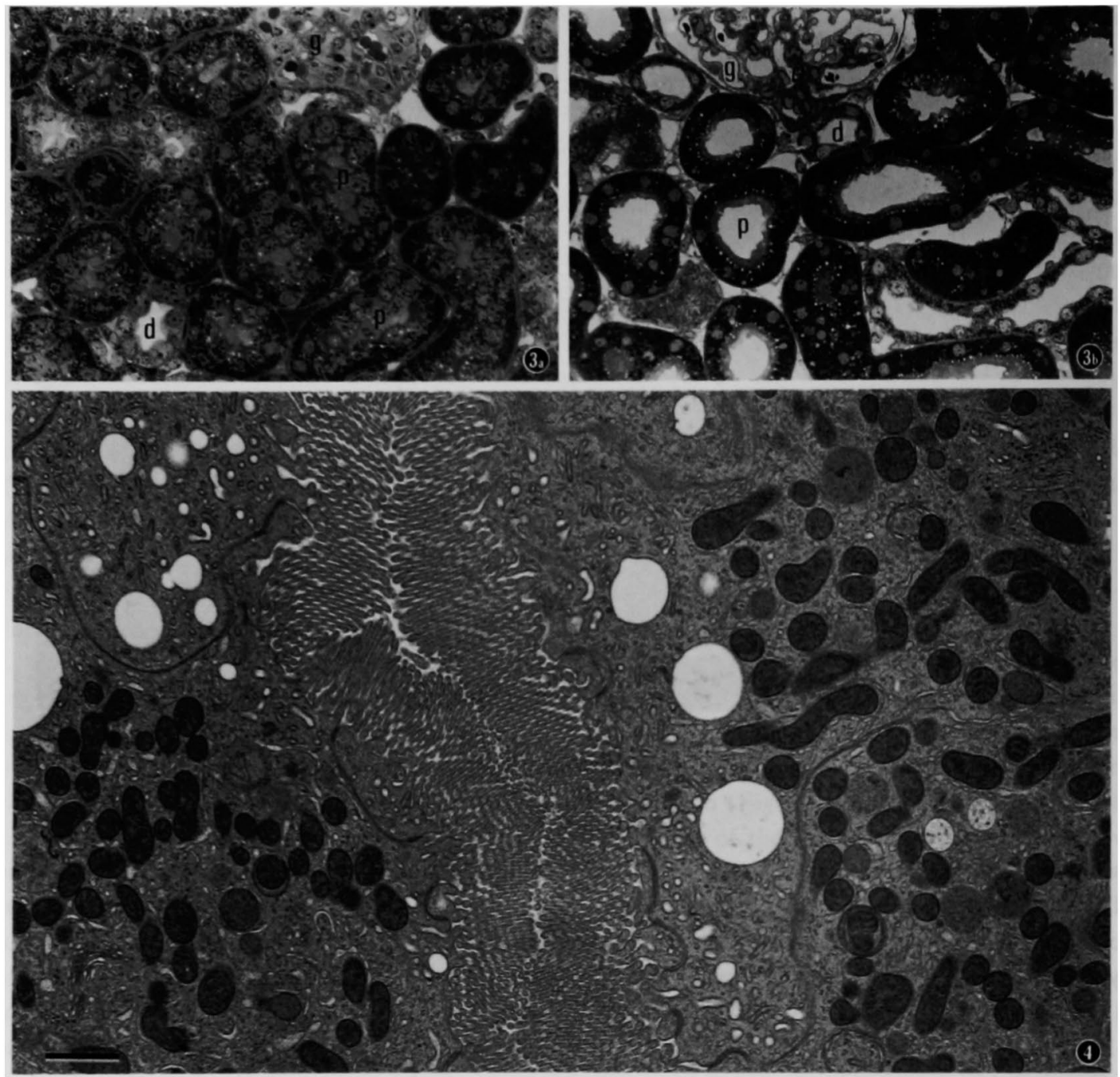

Fig. 3. Light micrographs of rat renal cortex. Semithick Epon-sections were stained with toluidine blue. d, distal renal tubule; g. glomerulus ; p. proximal convoluted tubule. (a) After immersion method of chemical fixation. The proximal tubule has an oocluded lumen filled with microvilli of brush borders. (b) After perfusion with glutaraldehyde-buffer solution. The proximal tubules appear to have a widely opened lumen.

Fig. 4. Luminal area of the proximal convoluted tubule of rat kidney processed by cryofixation. Tubular lumen is filled with numerous microvilli of brush border with a narrow interstice. $\times 9.500$.

lose the brush border and to attach with the plasma membrane of cells in the opposite side. Open appearance of the lumen in the materials processed by perfusion fixation, on the other hand, may be due to the result of shrinkage of the epithelial cells, because these cells appear much lower in height than those in other materials examined. The present observation indicates that the epithelial cells of the proximal convoluted tubule provided with brush border and closely apposed to each other with a narrow space may function more effectively for reabsorption of some proteins, amino acids, glucose, certain essential vitamins, and others in the glomerular filtrate.

Mucus-secreting cells: It is known that the goblet cells as well as mucous acinar cells of the salivary gland contain a number of secretory granules which occupy the major part of the cell body and appear to be swollen. This results in deformation of swollen cell body, disruption of the limiting membrane of individual secretory granules resembling fusion of granules and an irregularly- 


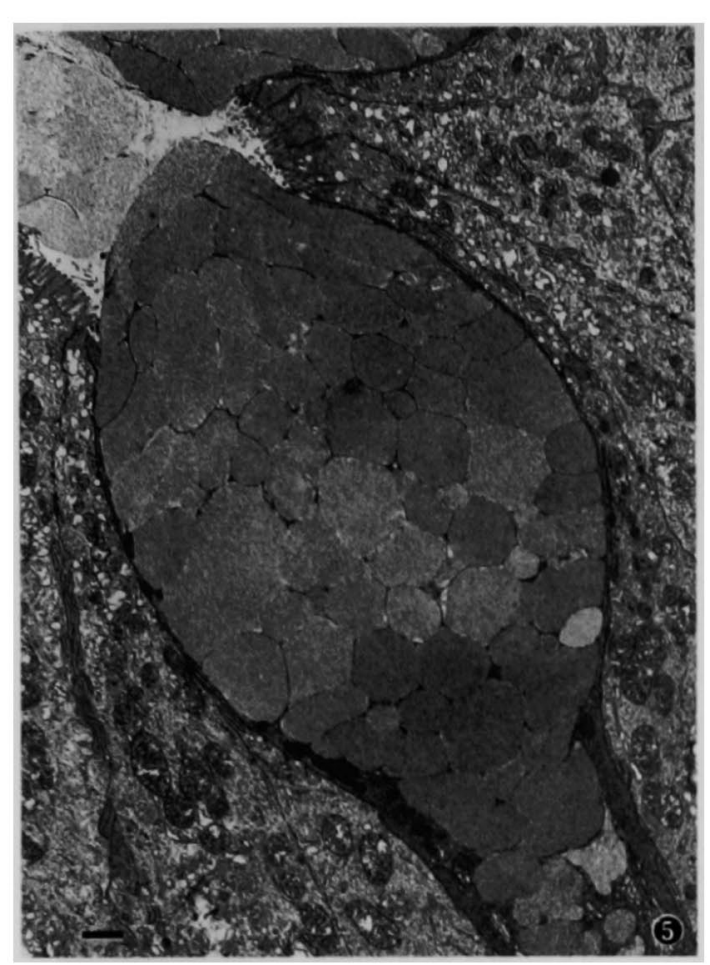

Fig. 5. Apical par of the goblet cell in rat colonic mucosa processed by conventional chemical fixation. Note the apical plasma membrane is lifted up into the lumen by swollen mucous secretory granules. The limiting membrane of individual granules is indistinct. $\times 4,200$.

shaped nucleus compressed toward the cell base (Fig. 5). Fragmentation of cell organelles such as Golgi stack, cisterns of rough-surfaced endoplasmic reticulum and mitochondria is also detected in many occasions. However, in those cells processed by the present cryofixation, secretory granules appear to be much smaller in size than those processed by chemical fixation and have a less dense homogeneous content surrounded with a distinct limiting membrane (Fig. 6a). Thus, the goblet cell does not show a goblet-like shape but has a columnar shape even in the storage phase of the secretory cycle. An oval nucleus is situated somewhat lower than the middle of the cell body. Parallel arrays of rough endoplasmic reticulum are developed well in the perinuclear and basal areas of the cytoplasm, and well-developed Golgi apparatus is observed as a continous entity with a cuplike shape in the supranuclear cytoplasm (For details, see ref. 2). Mucous acinar cells of the salivary gland are characterized with numerous secretory granules occupying the major portion of the apical cytoplasm and a flattened nucleus displaced toward the cell base in specimens processed by chemical fixation, while they appear to have a spherical nucleus slightly lower than the middle of the cell body, a number of secretory granules with a distinct limiting membrane in the apical cytoplasm, well-developed Golgi apparatus in the supranuclear area and well-developed parallel arrays of rough endoplasmic reticulum in perinuclear and basal regions of the cytoplasm after primary cryofixation (Fig. 7). Such cytological characteristics are basically similar to those of the serous acinar cell. Discharge of mucous secretory granules is carried out by exocytosis with the same way as in the serous ones. In the so-called "goblet cell", mucous secretory droplets are sequentially released by exocytosis to form a deep invagination of the surface plasma membrane (Figs. 6a, 6b, and 6c). Discharge of secrete is accelerated by formation of a large secretory droplet which oocurs by fusion of individual secretory granules with each other during the storage phase ${ }^{2}$. In traditional histology, ultrastructural analyses of secretory process in mucus-secreting cells were hardly done because of their largely modified cytoarchitecture owing to swelling of secretory material caused by specimen preparation. However, the present cryofixation method allowed us to disolve this problem. It is now clarified that the secretory process in the mucus-secreting cells is essentially the same as in the serous cells.

Fusion and fission of biological membranes: In materials processed by conventional chemical fixation, membrane event during exocytotic discharge of secretory droplets or fusion secretory granules during storage appears to be initiated by close contact of two membranes to form a pentalaminar structure. Then, the pentalaminar membrane converts to be trilaminar in appearance and disintegrated (Fig. 8a). Such an observation is detected in many occasions ${ }^{13.14}$, and this has been thought to indicate the mechanism of fusion and fission of membranes. However, in cryofixed materials, closely-apposed membranes are frequently observed to form a pentalaminar profile of fused membranes (Fig. 8b), and conversion of pentalaminar structure to trilaminar one is hardly 

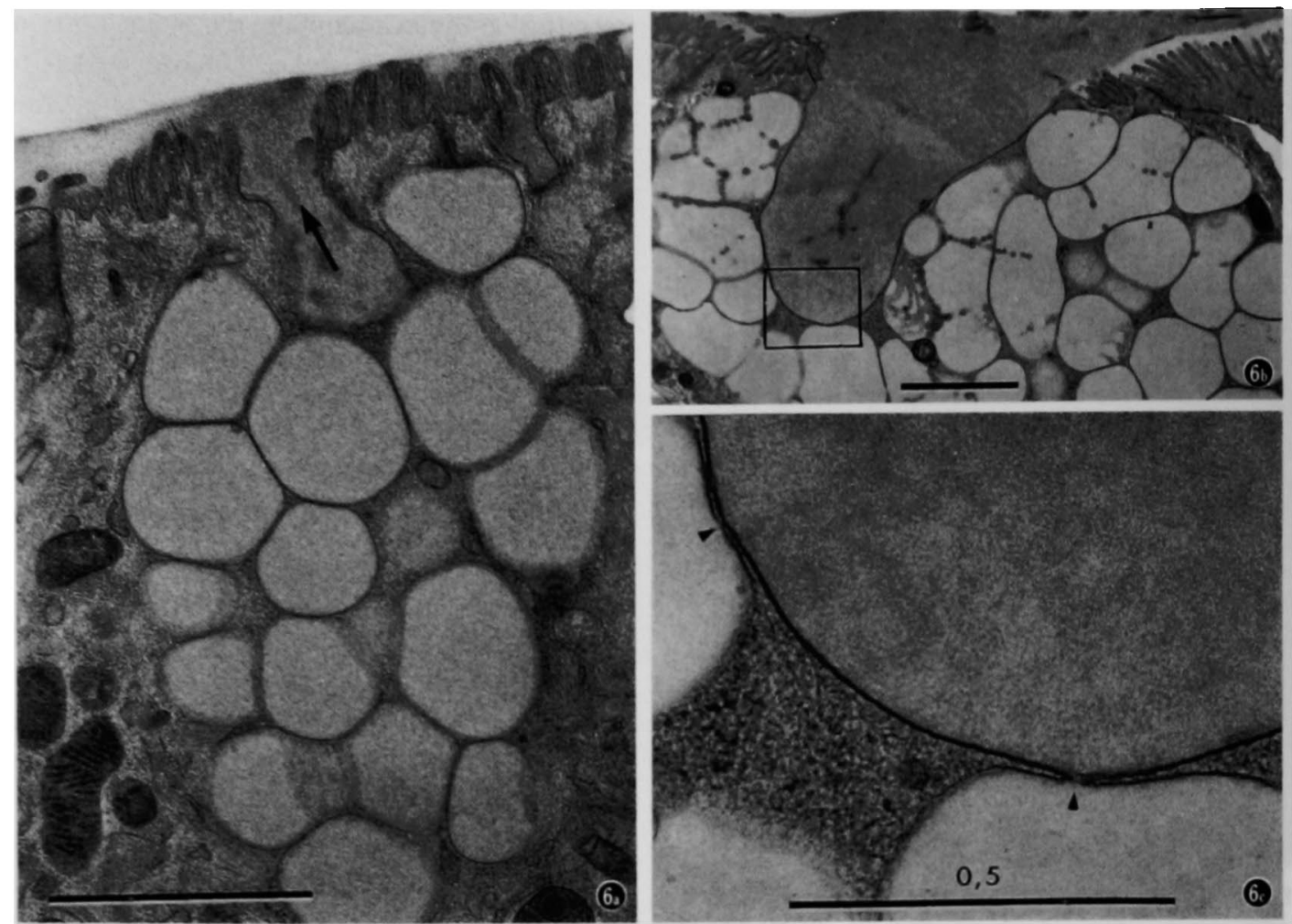

Fig. 6. Exocytotic release of secretory granules in the goblet cell processed by cryofixation. (a) A single secretory droplet is released by exocytosis in the initial stage of release. $\times 29,500$. (b) Sequential release of secretory droplets causes a deep invagination of the surface plasma membrane. Some droplets appear to fuse with each other to form a larger droplet before release. $\times 22,800$. (c) Framed area of Fig. 6b in higher magnification. Arrowheads indicate a small hole formed by fusion and fission between invaginated plasma membrane and the limiting membrane of secretory droplet. $\times \mathbf{8 0 . 8 0 0}$

observed even just before fission of fused membranes. Ornberg and Reeses have described a similar observation in amoebocyte processed by quick freezing and freeze-substitution. Thus, it may be concluded that the traditional observation of fusion and fission of membranes is an antifactitious appearance caused during specimen preparation.

\section{Ultrastructural features of intracytoplasmic} organelles: Protein-secreting cells are known to have well-developed parallel arrays of rough-surfaced endoplasmic reticulum in the perinuclear and basal regions of the cytoplasm. It is generally accepted that individual cisterns of the reticulum may change their morphology depending on their functional phases, that is they are dilated irregularly in an actively synthetic phase, although their lumen appears always to be empty regardless of their functional phase. Meanwhile, in the tissue processed by primary cryofixation, they are composed of long. continuous profiles of cisterns which are arranged in parallel to each other, and dilation of individual cisterns is rarely seen even in the synthetic stage after depletion of secretory granules (Figs. 9 and 10). Each cistern contains more or less amount of a moderately dense material with a finely granulated texture, and its interior appears to be higher in density than the cytoplasmic matrix (Figs. 10 and 11a). These findings represent well preservation of cisternal contents. It seems likely that irregularly dilated and/or fragmented profiles of cisterns of the reticulum do not reflect actively synthetic function of the reticulum but may be artifacts caused by poor preservation with preparatory procedures employed. In the pancreatic acinar cells $60 \mathrm{~min}$ after refeeding after fasting overnight, coated buds of ER cistern indicating transitional vesicle for- 




Fig. 7. Part of the serous demilune of mouse sublingual gland processed by cryofixation. Note that the mucous acinar cells have a spherical nucleus and a number of secretory granules surrounded with a limiting membrane $(\mathrm{m})$, while the serous acinar cell contains a number of secretory granules with a dense content (s) and a well-developed lamellar arrays of rough-surfaced endoplasmic reticulum. Arrows indicate a well-developed Golgi apparatus. $\times 6,200$.

mation are observed at the transitional area between rER and Golgi apparatus (Fig. Ila). Such an observation is detected more frequently in cryofixed materials than in chemically fixed materials. This indicates that cryofixation method is superior to chemical fixation to capture short-term events occurring in the cytoplasm.

In exocrine glandular cells examined in this study, including pancreatic acinar cells, serous and mucous acinar cells of the salivary glands and mucous-secreting cells in the colonic mucosa, the Golgi apparatus is developed well in the supranuclear cytoplasm and composed of a stack of flat cisterns and associated smalll vesicles. Several vacuoles with various sizes are found at the inside of concaved Golgi stack and some of them are occasionally seen to continue to the periphery of two or three innermost profiles of stacked cisterns. One of two outermost cisterns at the convex face have a wide lumen with bead-like constrictions. In materials processed by chemical fixation, all of these stacked cisterns appear to have a clear lumen which looks like empty. Stacked citserns tend to be fragmented into several groups in these samples. While, in cryofixed materials, the Golgi stack is preserved well and characterized with four to six layers of stacked flat cisterns in protein-secreting cells (Fig. 10), and eight to ten or more layers of stacked cisterns in mucus-secreting cells ${ }^{3}$. Individual cisterns as well as condensing vacuoles contain moderately dense material which increases gradually in density from the cis-side to the transside, but one or two outermost cisterns appear to be empty. Such observation may reflect the molecular density of material contained within the cisterns, because if the section is stained with PATSC-SP method for sugar histochemistry reaction product increases in density from cis-side to trans- 

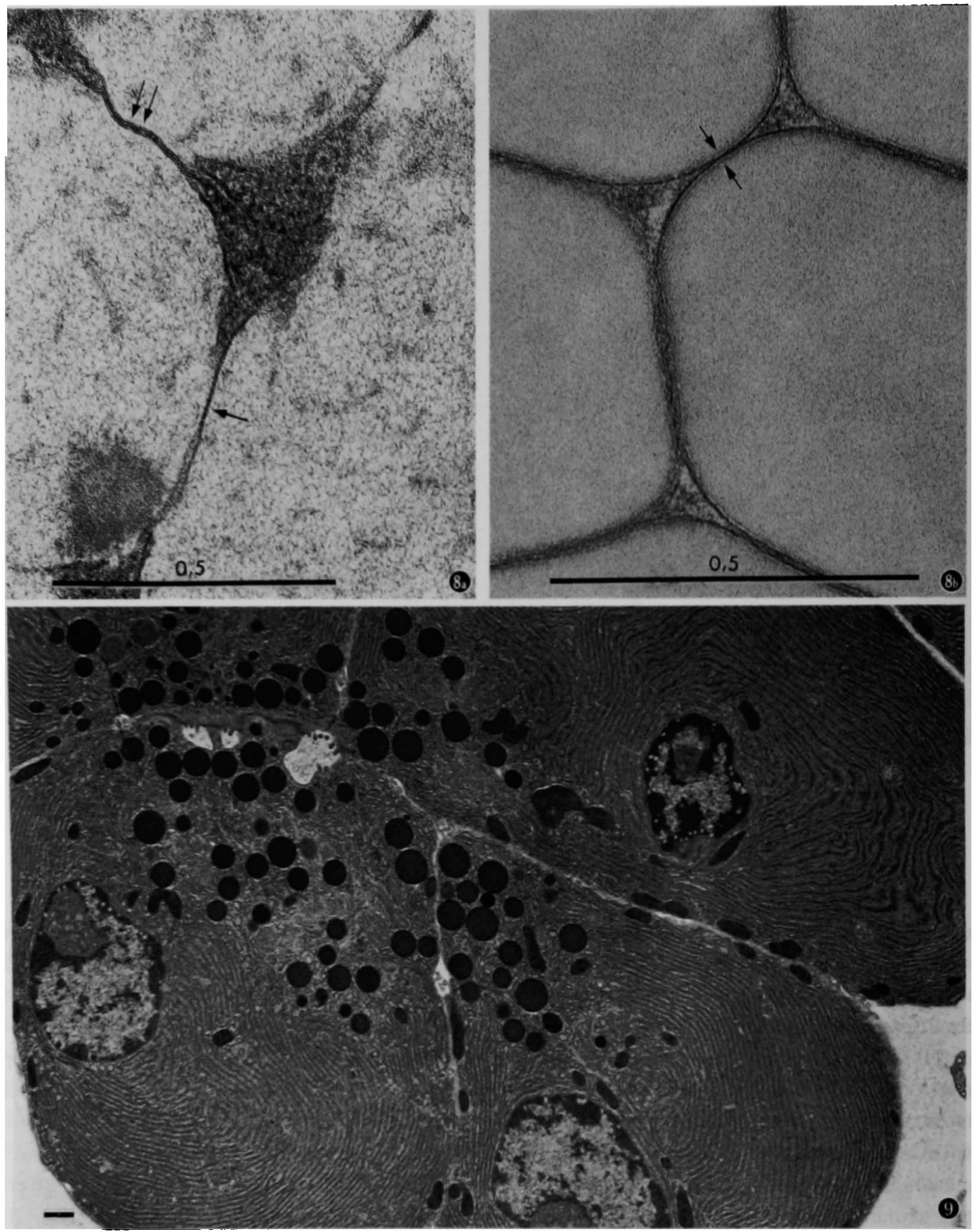

Fig. 8. Fusion of two membranes just before fission in secretory droplets of goblet cell. (a) Conversion of the pentalaminar structure of closely apposed limiting membranes (double arrow) to the trilaminar structure (single arrow), as seen in the materials processed by conventional chemical fixation. $\times 83,000$. (b) Pentalamellar structure of fused membranes (arrows) seen in the cryofixed material. Its conversion to the trilamellar one is hardly detected even just before fission of fused membranes. $\times 104,500$.

Fig. 9. Parallel arrays of rough-surfaced endoplamsic reticulum in mouse pancreatic acinar cells processed by cryofixation. Note that individual cisterns appear to be continuous and never be dilated irregularly regardless of functional phases of the gland. $\times 4,300$. 

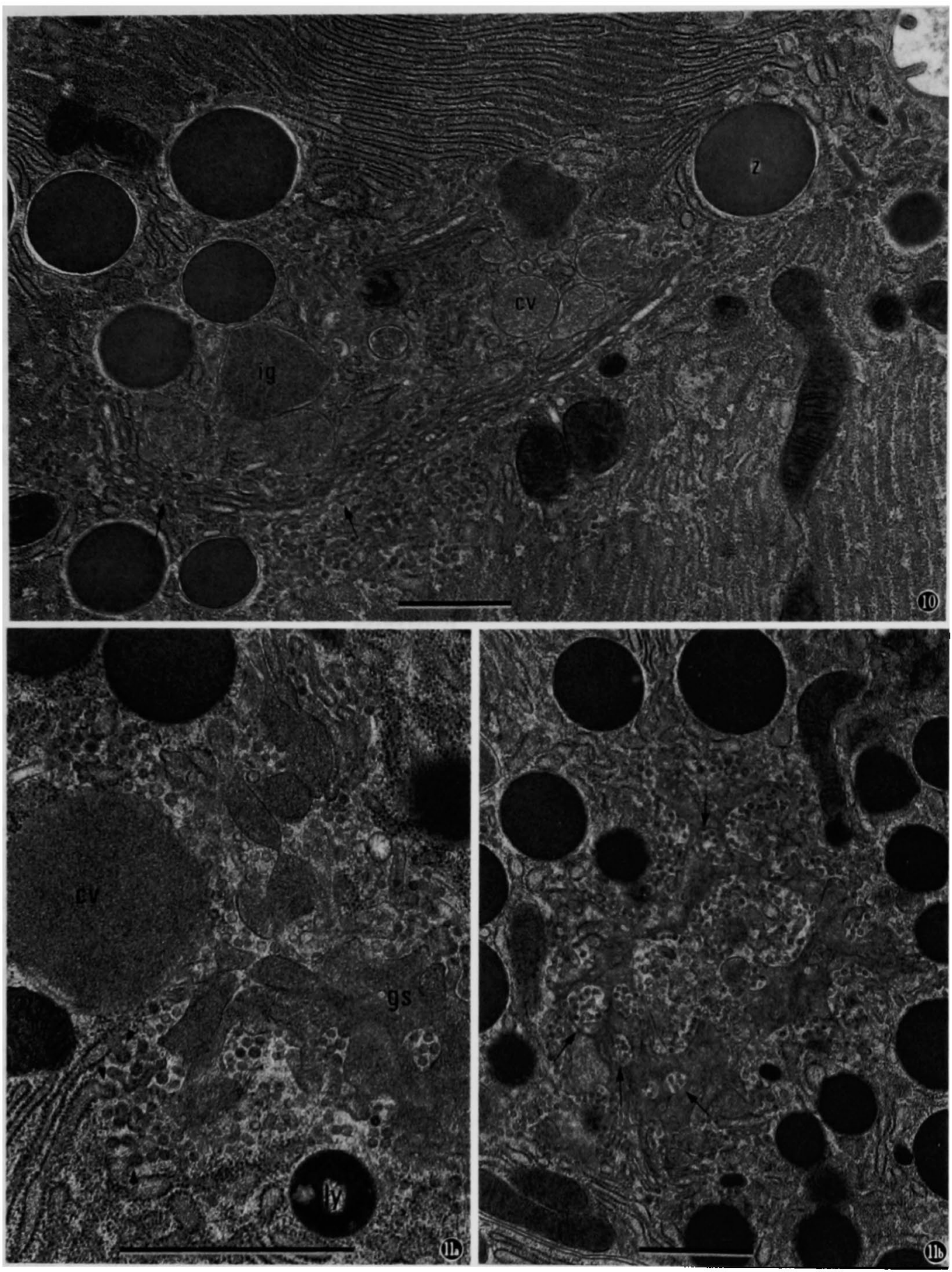

Fig. 10. Golgi apparatus of mouse pancreatic acinar cell $60 \mathrm{~min}$ after refeeding after fasting for 2 days. Arrows indicate discontinuities of the Golgi stack. cv, condensing vacuole ; ig. immature granule : 2, zymogen granule. $\times 16,200$.

Fig. 11. Golgi apparatus of mouse pancreatic acinar cell in the same material as in Fig. 10. (a) Transitional area between endoplamic reticulum and Golgi apparatus. Arrowheads indicate budding of cisterns of endoplasmic reticulum to form transitional vesicles. cv, condensing vacuole; $\mathrm{gs}$, a tangentially sectioned area of Golgi cistern; ly, lysosome. $\times 34,200$. (b) A tangential section of Golgi stack. Arrows indicate fenestrations of the stack which correspond to discontinuous areas of cross-sectioned Golgi stack seen in Fig. 10. $\times 16,200$. 
sides. Discontinuittes of the stack are seen frequently in cross-sectioned area of the apparatus, and numerous small vesicles accumulate around discontinuous regions (Fig. 10), while in tangentially sectioned area of the Golgi stack individual flat cisterns have a number of oval or round fenestrae, 100 to $500 \mathrm{~nm}$ or more in diameter, which are aligned to form cytoplasmic channels extending across the whole Golgi stack (arrows in Fig. 11 b). It seems likely that some small vesicles fuse with or bud off from the peripheral rim and perifenestral segments of individual cisterns (Fig. 11b). Three-dimensional observations of the Golgi stack using semithick sections revealed that the Golgi apparatus is composed of an irregularly undulated, stacked cisterns and associated small vesicles. and the cytoplasmic channels penetrating the stack provide pathways for the traffic of small vesicles ${ }^{3.15}$. Such profiles of the stacked cisterns and associated vesicles seem to be a common feature of the apparatus as seen in a wide variety of cells (unpublished data). In previous studies, the route of traffic of newly synthesized material processed within the Golgi apparatus remained unclear. However, the present observation provides a morphological evidence for structural organization of the traffic system in the Golgi apparatus, and also provides a reasonable interpretation for the processing and sorting mechanism of the materials in the apparatus. Thus, fragmentation and irregular dilation of the stacked cisterns as seen in traditional histology are thought to be an artifactitious alteration due to preparatory procedures. Another problem is concerned to an appearnace of the so-called "dark cells" in some epithelial tissues. Reality or arifact of the observation has been discussed for a long time, but it is now considered to be an artifact in most occasions ${ }^{16}$.

In conclusion, it is now clear that conventional chemical fixation may cause a variety of artifacts more frequently and seriously than we have thought. For routine works of pathological examination at the light microscopic level, these problems may not affect so seriously on precise diagnosis of histological changes in the sample. However, in ultrastructural or molecular analyses of samples, much attention should be paid to these problems, and one should keep in mind the limitation of interpretation to any observation obtained. Meanwhile, cryofixation method can be applied to certain materials only, because acceptable areas without disturbing ice crystal formation are limited to be a narrow area 10 to $20 \mu \mathrm{m}$ thick under the surface of the sample, which was frozen first be contact with the cooled metal mirror. Perfusion fixation is applicable to examination of small animals but not suitable for human materials and other samples from big animals. Thus, if more strict and precise diagnosis is required, it might be better to fix the samples with different fixatives more than one and compare the results obtained to each other in order to confirm its reality or artifact.

Acknowledgment: This study was partly supported by a Grant-in-Aid for Scientific Research from the Ministry of Education, Science and Culture of Japan.

\section{References}

I. Ichikawa, A, Ichikawa, $\mathbf{M}$, and Hirokawa, $\mathbf{N}$ : The ultrastructure of rapid-frozen, substitution fixed parotid acinar cells of the Mongolian gerbli (Meriones unguiculatus). Am J Anat 157: 107-110, 1980.

2. Ichikawa, M. Ichikawa, A, and Kidokoro, S : Secretory process of mucus-secreting cells in mouse colonic mucosa studied by rapid freezing and freezesubstitution. J Eelectron Microsc 36: 117-127. 1987.

3. Ichikawa, $\mathbf{M}$ and Ichikawa, $\mathbf{A}$ : The fine structure of sublingual gland acinar cells of the Mongolian gerbil, Meriones unguiculatus, processed by rapid freezing followed by freeze-substitution fixation. Cell Tiss Res 250 : 305-314, 1987.

4. Heuser, JE, Reese, TS, Dennis, MI, Jan, Y, Jan L, and Evans, L: Synaptic vesicle exocytosis captured by quick freezing correlated with quantal transmitter release. J Cell Biol 81 : 275-300, 1979.

5. Ornberg. RL and Reese, TS: Beginning of exocylosis captured by rapid freezing of Limulus amebocytes. J Cell Biol 90: 40-54, 1981.

6. Ichikawa. M, Sasaki, K, and Ichikawa, A : Immunocytochemical localization of amylase in gerbil salivary gland acinar cells processed by rapid freezing and freeze-substitution fixation. $J$ Histochem Cytochem 37 : 185-194, 1989.

7. Ikeda, $\mathbf{H}$ : An ultrastructural analysis of the inclusion body in the type 11 pneumocyte processed by rapid freezing followed by freeze-substitution. An autoradiographic study. J Electron Microsc 34: 398-410, 1985.

8. Forssmann, GW, Ito, S, Weihe, E, Aoki, A, Dym, M, and Fawcett, DW: An improved perfusion fixation method for the testis. Anat Rec 188: 307-314, 
1977.

9. Petersen, $\mathrm{OH}$ : The Electrophysiology of Gland Cells. Academic Press, London, 1980.

10. Cardell, RR, Badenhausen, S, and Porter, KR : Intestinal absorption in the rat ; an electron microscopical study. J Cell Biol 34: 123-155. 1967.

11. Kaye, GI, Wheeler, HD, Whitlock, RT, and Lane, $\mathbf{N}$ : Fluid transport in rabbit gall bladder : a combined physiological and electron microscope study. J Cell Biol 30 : 237-268, 1966.

12. Maunsbach, AB: Observations on the segmentation of the proximal tubule of the rat kidney. J Ultrastruct Res 16: 239-254, 1966.

13. Palade, GE: Intracellular aspects of the pancreas of protein synthesis. Science 189: 347-358, 1975.

14. Tandler, $M$ and Poulsen, JH: Fusion of the envelop of mucous droplets with the luminal plasma membrane in acinar cells of cat submandibular gland. J Cell Biol 68: 775-781, 1976.

15. Ichikawa, $A$, Ichikawa, $M$, and Watabe, $T$ : High resolution analysis of three-dimensional structure of the Golgi apparatus in rapid-frozen, substitution fixed gerbil sublingual gland acinar cells. J Electron Microsc 31 : 397-401, 1982.

16. Ichikawa, A : A note on the "dark cells" of the exocrine pancreas. Arch Histol Jpn 28: 79-88, 1967. 